\title{
Use of CPAP Machines in the Perioperative Setting in Ambulatory Surgical Centers
}

\author{
Javier Marull ${ }^{1, *}$ \\ M Jonathan Vachon $\mathbb{D}^{1, *}$ \\ Dylan Buitran ${ }^{2}$ \\ Amy Macaluso' \\ 'University of Texas Southwestern \\ Medical Center, Dallas, TX, 75390, USA; \\ ${ }^{2}$ University of California Irvine, Irvine, \\ CA, 92697, USA
}

*These authors contributed equally to this work
Correspondence: Javier Marull

University of Texas Southwestern Medical

Center, 180I Inwood Road, 2nd Floor

Outpatient Surgery Center, Dallas, TX, 75390, USA

Tel + I 2|4-403-4592

Email javier.marull@utsouthwestern.edu
Introduction: Obstructive sleep apnea is a common sleep-related breathing disorder that is associated with significant perioperative complications. In 2012 and 2017, Society of Ambulatory Anesthesia and Society of Anesthesia and Sleep Medicine published consensus statements for the selection of patients with OSA scheduled for ambulatory surgery. Despite these recommendations, the need for a CPAP device in the immediate postoperative period at ambulatory surgical centers remains controversial because these ambulatory patients are healthier and have fewer complications. This study aims to investigate the compliance rate with this recommendation among busy ASCs.

Methods: We created a survey to investigate if ASCs require patients to bring their CPAP devices to the facility. The survey measured compliance rates of ASCs to SAMBA's recommended guidelines of having CPAP machines available.

Results: The survey had a response rate of 60.9\% encompassing 408,147 cases among 1946 providers. Of the facilities that responded, only $59.7 \%$ of them required their patients to bring their CPAP devices on the day of surgery. Out of the 67 facilities that responded, only $25.37 \%$ reported using a CPAP machine postoperatively within the past 2 years, with the highest CPAP usage at one facility being 20 times in that 2-year period.

Discussion: This would mean that $40.3 \%$ of ASCs that did respond do not have access to a CPAP device on-site and may possibly lack the proper equipment needed to handle these complications. The frequency and fatality rate associated with postoperative respiratory complications requiring a CPAP device are still inconclusive, making the need for CPAP devices during perioperative management controversial. Studies further in-depth are therefore necessary to assess postoperative complications that require the use of a CPAP device to determine the urgency of ASCs implementing SAMBA's recommendations.

Keywords: obstructive sleep apnea, perioperative care, postoperative care, CPAP, compliance

\section{Introduction}

Obstructive sleep apnea (OSA) plays a significant role in the planning of perioperative management of patients. OSA is characterized by recurrent episodes of partial (hypopnea) or complete (apnea) interruption in breathing during sleep, and symptoms include loud snoring, nocturnal awakening, and daytime sleepiness. ${ }^{1,2}$ Physically, patients with OSA commonly have a wider neck circumference and a smaller pharyngeal space, leading to airway obstruction that can pose a significant challenge in perioperative management. Its etiology is multifactorial, and management is predicated on the severity of obstruction.

OSA has been linked to causing certain cardiovascular diseases such as hypertension, coronary artery disease, and atrial fibrillation. Classically, OSA is treated 
with weight loss management and a Continuous Positive Airway Pressure (CPAP) machine, which provides a constant positive pressure through the pharyngeal space, stimulating muscle activity in the pharynx, allowing for more airflow. ${ }^{3}$ The risks associated with perioperative management of patients with OSA include ineffective mask ventilation and intubation, postoperative airway obstruction, and other complications resulting from comorbid conditions. ${ }^{4}$ Asphyxiation and aspiration are the major risks in the perioperative management of patients with OSA, as many medications used by anesthesiologists depress muscle activity and suppress arousal responses, resulting in the airway being inaccessible during operations. 5

In 2012 and 2017, SAMBA (Society of Ambulatory Anesthesia) and SASM (Society of Anesthesia and Sleep Medicine) published consensus statements for the selection of patients with OSA scheduled for ambulatory surgery. ${ }^{6,7}$ Despite these guidelines, the need for a CPAP device in the immediate postoperative period remains controversial. At many ASCs, CPAP devices are not currently being used because the patients at these facilities are healthier and usually have only mild-to-moderate OSA. Also, patients may forget to bring the CPAP device or it is not feasible for them to bring it for the same-day surgery. Many anesthesiologists have observed that the CPAP machines are not being used at the ASCs as recommended. Reasons for the noncompliance with the recommendations include the quick emergence and recovery of the patients for the faster day surgery cases, and these patients are only in the PACU for an average of 1 hour postoperatively at ASCs. This study aims to investigate the compliance rate with this recommendation among busy ambulatory surgery centers (ASC) who mainly have healthy patients with mild-to-moderate OSA or those with OSA and well-managed comorbidities, as the need for CPAP machines in the postoperative period at these ASCs remains unclear. To this end, we decided to conduct a survey of medical directors of ASCs throughout the United States (US).

\section{Methods}

We created a survey to investigate if the ASCs that accept OSA patients require the patients to bring their CPAP devices to the facility (Box 1). Additionally, we inquired if those ASCs used CPAP devices in the immediate postoperative period within the past 2 years. The survey was validated by five practitioners of busy ASCs to confirm
Box I Survey to Investigate if the ASCs That Accept OSA Patients Require Patients to Bring Their CPAP Devices to the Facility. Additionally, We Inquired if Those ASCs Used CPAP Devices in the Immediate Postoperative Period Within the Past 2 Years

\begin{tabular}{|l|}
\hline Questions Asked in the Survey to the ASCs \\
\hline Number of surgical procedures per year. \\
Number of anesthesiologists/anesthesia providers. \\
Do you have patients with OSA in your facility? If yes, please proceed \\
with the next question. \\
Do you ask your patients to bring their CPAP machine to the ASC? If \\
not, why? \\
How often have you had to use a CPAP machine in your facility in the \\
past 2 years?
\end{tabular}

whether any of the questions were difficult to understand or to answer via email, and a reminder email was sent if no response was received within a month. The results of the survey were analyzed using spreadsheets and presented as percentages.

\section{Results}

The survey had a response rate of $60.9 \%$ (67 of 110 ASC medical directors that submitted the survey), encompassing 408,147 cases among 1946 providers. Of the facilities that responded, only $59.7 \%$ of them required their patients to bring their CPAP devices on the day of surgery. Out of the 67 facilities that responded, only $25.37 \%$ (17/67) reported using a CPAP machine postoperatively within the past 2 years, with the highest CPAP usage being 20 times at one facility during that 2 -year period.

\section{Discussion/Conclusion}

Obstructive Sleep Apnea is a respiratory condition that increases the risk of $\mathrm{CV}$ disease, notably atrial fibrillation, hypertension, and coronary artery disease. Perioperatively, it also increases the risk for ineffective mask ventilation and intubation, postoperative airway obstruction, asphyxiation, and aspiration. In 2012 and 2017, SAMBA (Society of Ambulatory Anesthesia) and SASM (Society of Anesthesia and Sleep Medicine) published consensus statements for the selection of patients with OSA scheduled for ambulatory surgery. ${ }^{6,7}$ The recommendations include:

1. Patients with a known diagnosis of OSA and optimized comorbid medical conditions can be 
considered for ambulatory surgery if they are able to use the CPAP device in the postoperative period.

2. Patients with presumed diagnosis of OSA, based on screening tools such as the STOP-Bang questionnaire and optimized comorbid conditions can be considered for ambulatory surgery if postoperative pain can be managed predominantly with nonopioid analgesic techniques. It is not necessary to postpone surgery in this patient population.

3. OSA patients with non-optimized comorbid conditions may not be ideal candidates for ambulatory surgery.

4. Patients receiving preoperative CPAP should be instructed to bring their CPAP device to the ambulatory care facility unless one is available at the facility.

5. Continued use of PAP therapy at previously prescribed settings is recommended during periods of sleep, both preoperatively and postoperatively. Adjustments may need to be made to the settings to account for perioperative changes such as facial swelling, fluid shifts, recent pharmacotherapy, and pulmonary function.

The survey we conducted showed that $59.7 \%$ of the ASC facilities (40) that responded adhered to SAMBA's recommendations, having access to CPAP devices on-site to treat immediate postoperative complications in patients with OSA (Box 1). This would mean that $40.3 \%$ of ASCs (27/40) that did respond do not have access to a CPAP device on-site and, as a result, may possibly lack the proper equipment needed to handle these complications. However, approximately $75 \%$ of the ASCs that followed SAMBA's recommendations by having CPAP available did not actually use the CPAP device postoperatively within the last two years. The highest reported CPAP usage rate was only 20 times in that 2-year period at one facility. The frequency and fatality rate associated with postoperative respiratory complications requiring a CPAP device at ASCs with healthier OSA patients are still inconclusive, making the need for CPAP devices during perioperative management at these facilities controversial. Therefore, more in-depth studies are necessary to assess postoperative complications that require the use of a CPAP device in order to determine the urgency of ASCs implementing SAMBA's recommendations. A proposal for correction of this problem of not using

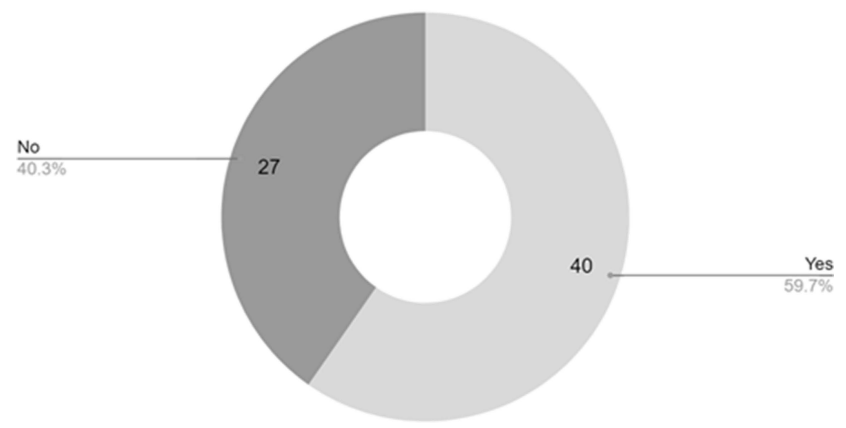

Figure I ASC's compliance with SAMBA recommendations for perioperative management of patients with OSA.

the CPAP devices at the ASCs as recommended could include the following:

(a) Bring a permanent hospital CPAP and get approval by Biomed,

(b) Train nurses how to use the CPAP device,

(c) Assign PACU nurses one to one with OSA patients,

(d) Keep OSA patients in PACU longer.

Other considerations for future OSA studies for postoperative treatment will also need to include the new hypoglossal nerve stimulator devices (Inspire) as treatment considerations for OSA in the perioperative period.

Compliance rate of ASCs that responded to the survey of SAMBA's recommendations for the perioperative management of patients with OSA (Figure 1).

\section{Ethics Statement}

Approval was not required for this research by an institutional review board or ethics committee due to a survey that was sent to medical directors of ambulatory surgery centers and reviews of medical charts were not necessary.

\section{Acknowledgments}

Contents have not been published elsewhere, and the paper is not being submitted elsewhere.

\section{Disclosure}

The authors report no conflicts of interest in this work.

\section{References}

1. De Backer W. Obstructive sleep apnea/hypopnea syndrome. Panminerva Med. 2013;55(2):191-195.

2. Patel SR. Obstructive sleep apnea. Ann Intern Med. 2019;171(11): ITC81-ITC96. doi:10.7326/AITC201912030 
3. Chowdhury O, Wedderburn CJ, Duffy D, Greenough A. CPAP review. Eur J Pediatr. 2012;171(10):1441-1448. doi:10.1007/ s00431-011-1648-6

4. Fassbender P, Herbstreit F, Eikermann M, Teschler H, Peters J. Obstructive sleep apnea-a perioperative risk factor. Dtsch Arztebl Int. 2016;113(27-28):463-469. doi:10.3238/arztebl.2016.0463

5. Hillman DR, Loadsman JA, Platt PR, Eastwood PR. Obstructive sleep apnea and anaesthesia. Sleep Med Rev. 2004;8(6):459-471. doi:10.1016/j.smrv.2004.07.002
6. Joshi GP, Ankichetty SP, Gan TJ, Chung F. Society for ambulatory anesthesia consensus statement on preoperative selection of adult patients with obstructive sleep apnea scheduled for ambulatory surgery. Anesth Analg. 2012;115(5):1060-1068. doi:10.1213/ ANE.0b013e318269cfd7

7. Chung F, Joshi GP. Preoperative screening and assessment of patients with obstructive sleep apnea: perspectives from ASA, SAMBA and SASM. ASA Monit. 2017;81:10-12.

\section{Publish your work in this journal}

Nature and Science of Sleep is an international, peer-reviewed, open access journal covering all aspects of sleep science and sleep medicine, including the neurophysiology and functions of sleep, the genetics of sleep, sleep and society, biological rhythms, dreaming, sleep disorders and therapy, and strategies to optimize healthy sleep.
The manuscript management system is completely online and includes a very quick and fair peer-review system, which is all easy to use. Visit http://www.dovepress.com/testimonials.php to read real quotes from published authors. 